\title{
All-optical polarization and amplitude modulation of second-harmonic generation in atomically thin semiconductors
}

\author{
Sebastian Klimmer ${ }^{1}$ 1, Omid Ghaebi', Ziyang Gan², Antony George ${ }^{2,3}$, Andrey Turchanin ${ }^{2,3}$, \\ Giulio Cerullo ${ }^{4}$ and Giancarlo Soavi ${ }^{1,3}$ 厌
}

\begin{abstract}
Second-harmonic generation is of paramount importance in several fields of science and technology, including frequency conversion, self-referencing of frequency combs, nonlinear spectroscopy and pulse characterization. Advanced functionalities are enabled by modulation of the harmonic generation efficiency, which can be achieved with electrical or all-optical triggers. Electrical control of the harmonic generation efficiency offers large modulation depth at the cost of low switching speed, by contrast to all-optical nonlinear devices, which provide high speed and low modulation depth. Here we demonstrate all-optical modulation of second-harmonic generation in $\mathrm{MoS}_{2}$ with a modulation depth of close to $100 \%$ and speed limited only by the fundamental pulse duration. This result arises from a combination of $D_{3 h}$ crystal symmetry and the deep subwavelength thickness of the sample, it can therefore be extended to the whole family of transition metal dichalcogenides to provide great flexibility in the design of advanced nonlinear optical devices such as high-speed integrated frequency converters, broadband autocorrelators for ultrashort pulse characterization, and tunable nanoscale holograms.
\end{abstract}

S temming from the first demonstration of optical harmonic generation $^{1}$, nonlinear optics has been in the spotlight of science and technology for more than half a century. In particular, second-harmonic generation (SHG) is a second-order nonlinear process widely used for frequency conversion, self-referencing of frequency combs ${ }^{2}$, crystal symmetry and Rashba effect studies ${ }^{3,4}$, sensing ${ }^{5}$, interface spectroscopy ${ }^{6}$ and ultrashort pulse characteriza$\operatorname{tion}^{7}$. Aside from free-space applications, there is increasing interest towards the realization of microscale integrated nonlinear devices. Here, a major challenge comes from the centrosymmetric nature of silicon $(\mathrm{Si})$ and silicon nitride $\left(\mathrm{Si}_{3} \mathrm{~N}_{4}\right)$, which forbids second-order nonlinearities. Large efforts have been devoted to the integration of nonlinear crystals such as lithium niobate ${ }^{8,9}$, or to symmetry breaking in $\mathrm{Si}$ and $\mathrm{Si}_{3} \mathrm{~N}_{4}$, for instance, via strain ${ }^{10}$, electric fields ${ }^{11}$ or the photogalvanic effect ${ }^{12}$.

Two-dimensional materials such as graphene and transition metal dichalcogenides (TMDs) hold great promise for nonlinear optical applications. They have a strong and broadband optical response $\mathrm{e}^{13,14}$, combined with the possibility of harmonic generation enhancement at excitonic resonances in TMDs ${ }^{15}$ and at multiphoton resonances in graphene's Dirac cones ${ }^{16,17}$. Furthermore, thanks to their flexibility and mechanical strength ${ }^{18}$, they can be easily integrated into photonic platforms. Various functionalized devices for sensing and frequency conversion have been demonstrated on fibres ${ }^{19}$, waveguides ${ }^{20}$ and microring $s^{21}$, while direct patterning of TMDs has been used to realize atomically thin meta-lenses ${ }^{22,23}$ and nonlinear holograms ${ }^{24,25}$. Furthermore, harmonic generation in two-dimensional materials can be efficiently tuned by external electrical ${ }^{16,26-28}$ or all-optical excitation ${ }^{29,30}$, offering an extra degree of freedom for the design of advanced nanoscale devices.
However, all of the electrical and all-optical schemes that have been proposed so far for SHG modulation in two-dimensional materials have considerable downsides. On one hand, electrical modulation has been demonstrated in tungsten diselenide ( $\left.\mathrm{WSe}_{2}\right)$ monolayers ${ }^{26}$ by tuning the oscillator strength of neutral and charged exciton resonances through electrostatic doping, and also in molybdenum disulfide $\left(\mathrm{MoS}_{2}\right)$ homobilayers ${ }^{27}$ by breaking the naturally occurring inversion symmetry through electrical gating, in the latter case with a large modulation depth of up to a factor of 60; however, electronics is intrinsically slower than optics and photonics. On the other hand, all-optical SHG modulation has been achieved by quenching of the exciton oscillator strength following ultrafast optical excitation in $\mathrm{MoS}_{2}$ (refs. ${ }^{29,30}$ ). This approach offers high modulation speed and is limited in principle only by the excited state/exciton lifetime (approximately tens of picoseconds); however, the largest depth in all-optical SHG modulation reported ${ }^{29}$ so far in TMDs is $55 \%$, with a strong dependence on the excitation wavelength and fluence. Furthermore, this scheme for all-optical SHG modulation is only effective for excitation and frequency conversion above-gap or at excitonic resonances and it is not applicable for below-gap excitation, thus leading to a naturally limited spectral bandwidth.

Here we demonstrate a novel approach for the all-optical control of the second-harmonic (SH) polarization in $\mathrm{MoS}_{2}$ and show that this can be used for all-optical modulation of the SH efficiency with modulation depth close to $100 \%$ and speed limited only by the fundamental frequency (FF) pulse duration. Our method relies solely on symmetry considerations in combination with the deep subwavelength thickness of the sample and thus does not require resonant enhancement or above-gap excitation for its implementation. Moreover, the same approach can be extended to any

'Institute of Solid State Physics, Friedrich Schiller University Jena, Jena, Germany. ${ }^{2}$ Institute of Physical Chemistry, Friedrich Schiller University Jena, Jena, Germany. ${ }^{3}$ Abbe Center of Photonics, Friedrich Schiller University Jena, Jena, Germany. ${ }^{4}$ Dipartimento di Fisica, Politecnico di Milano, Milan, Italy.

凶e-mail: giancarlo.soavi@uni-jena.de 

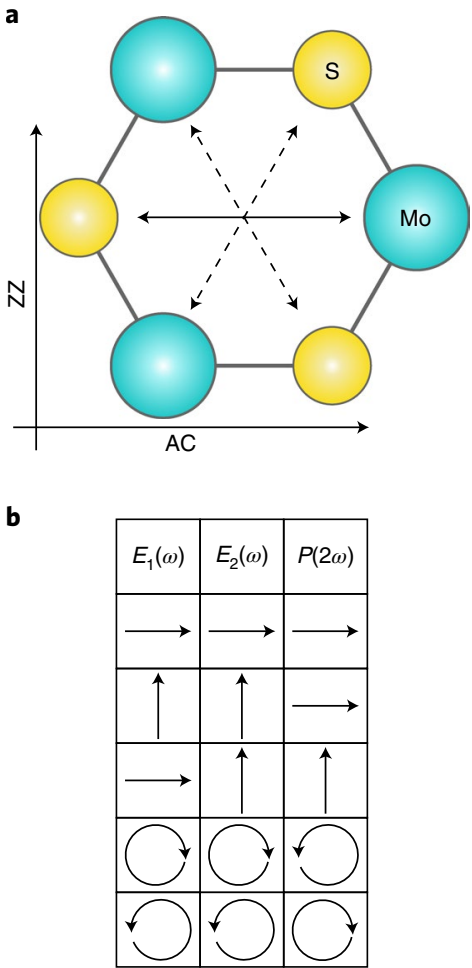

c

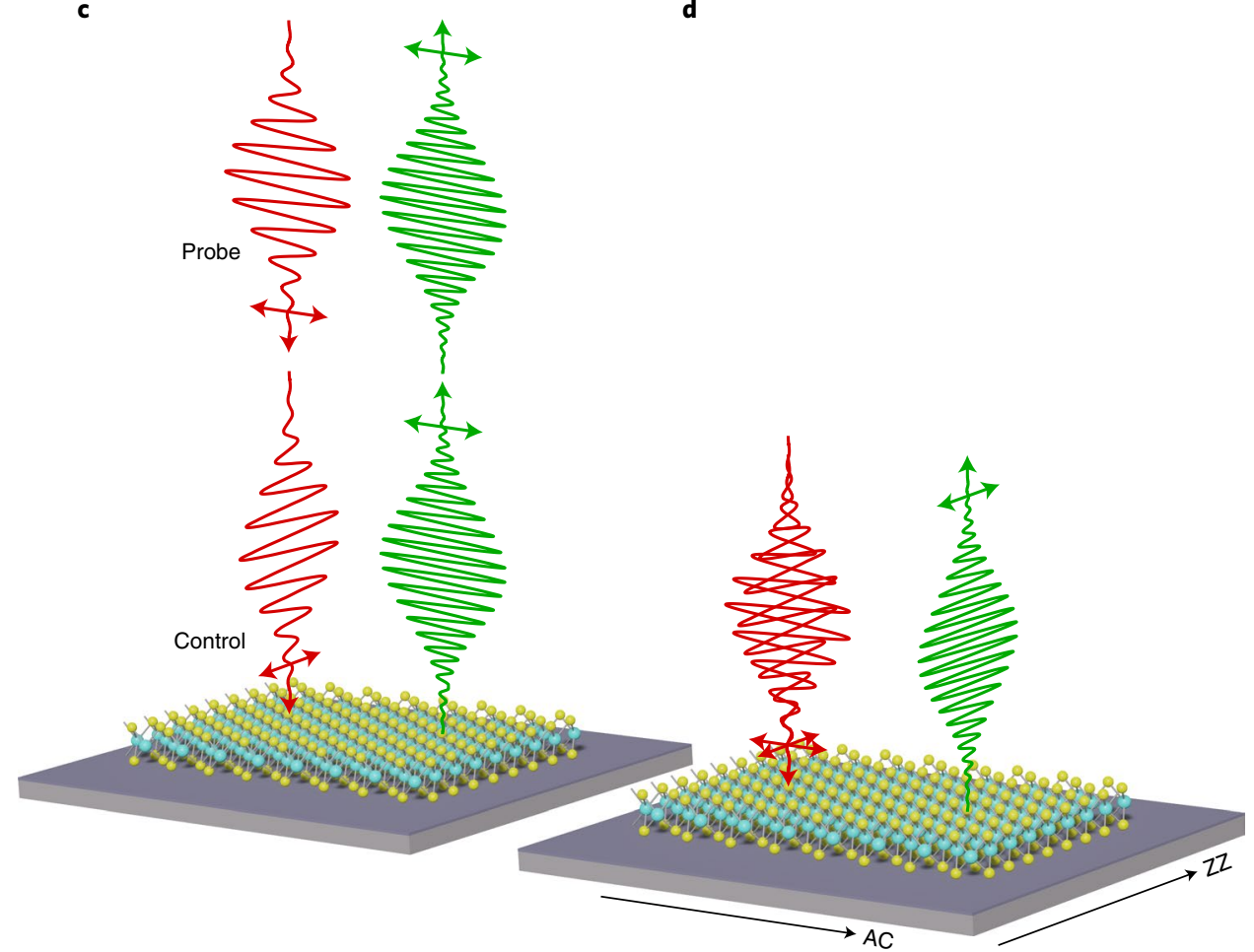

Fig. 1 | $\mathrm{MoS}_{2}$ symmetry properties, optical selection rules and an all-optical SHG modulation scheme. a, Top view of a MoS $\mathrm{crystal}$ The arrows inside the hexagon highlight the $D_{3 h}$ three-fold rotational symmetry. $\mathbf{b}, \mathrm{A}$ schematic of the resulting SH polarization for different combinations of FFs along the AC (horizontal arrows) and ZZ (vertical arrows) directions. c,d, A sketch of the all-optical SH polarization modulation. The control pulse is polarized along the $\mathrm{ZZ}$ direction whereas the probe pulse is polarized along the AC direction of the $\mathrm{MoS}_{2}$ sample. $\mathbf{c}$, When the delay between the control and probe pulses is larger than the FF pulse duration, both will generate a SH signal polarized along the AC direction. $\mathbf{d}$, For zero delay between the probe and control pulses the $\mathrm{SH}$ signal will be emitted along the ZZ direction.

two-dimensional material belonging to the $\mathrm{D}_{3 h}$ symmetry group, thus for instance to any material of the TMD family. Our findings provide a new strategy for the tuning of the fundamental properties of light (polarization and amplitude) in the nonlinear regime and in the two-dimensional thickness limit, and thus pave the way to the design of novel advanced functionalities in high-speed frequency converters, nonlinear all-optical modulators and transistors ${ }^{31,32}$, interferometric autocorrelators for ultrashort pulse characterization and tunable atomically thin holograms ${ }^{24}$.

\section{Nonlinear optical characterization}

For the experiments, we used high-quality monolayer $\mathrm{MoS}_{2}$ flakes fabricated by a modified chemical vapour deposition method $^{33,34}$ on thermally oxidized $\mathrm{Si} / \mathrm{SiO}_{2}$ substrates. By contrast to thin films where surface SHG is allowed due to an out-of-plane component of the second-order susceptibility ${ }^{6,35}$, TMDs belong to the $D_{3 h}$ symmetry group and thus have only one non-vanishing in-plane component of the nonlinear optical susceptibility (Methods) ${ }^{35-40}$

$$
\chi^{(2)} \equiv \chi_{y y y}^{(2)}=-\chi_{y x x}^{(2)}=-\chi_{x y x}^{(2)}=-\chi_{x x y}^{(2)},
$$

where $x$ and $y$ refer to the in-plane Cartesian coordinates of the $\mathrm{SH}$ polarization and of the two FFs. A sketch of the hexagonal lattice for $\mathrm{MoS}_{2}$ is shown in Fig. 1a, in which the Cartesian coordinates are defined with respect to the two main lattice orientations: the armchair (AC) and zigzag (ZZ) directions. In this framework, the SH intensity $I^{\omega}$ as a function of the FF for any TMD along the AC and $\mathrm{ZZ}$ directions can be written as

$$
I_{\mathrm{AC}}^{2 \omega} \propto\left|E_{\mathrm{AC}}^{2}-E_{\mathrm{ZZ}}^{2}\right|^{2},
$$

$$
I_{\mathrm{ZZ}}^{2 \omega} \propto\left|2 E_{\mathrm{AC}} E_{\mathrm{ZZ}}\right|^{2},
$$

where $E_{\mathrm{AC}}$ and $E_{\mathrm{ZZ}}$ correspond to the FF fields with polarization along the $\mathrm{AC}$ and $\mathrm{ZZ}$ directions, respectively ${ }^{39,41-43}$. The SHG from two electric fields with the same polarization (either along $\mathrm{AC}$ or $\mathrm{ZZ}$ ) will thus always result in an emitted $\mathrm{SH}$ intensity with polarization along the AC direction, as depicted in Fig. 1 b. This is indeed the case for all of the SHG experiments on two-dimensional materials performed so $\operatorname{far}^{15,36,39,43}$. On the other hand, two ultrashort FFs with perpendicular polarization (along the $\mathrm{AC}$ and $\mathrm{ZZ}$ directions) and with the same amplitude will generate a $\mathrm{SH}$ signal along the $\mathrm{AC}$ direction if they do not overlap in time (Fig. 1c), whereas they will generate a SH signal along the ZZ direction at zero delay (Fig. 1d), thus leading to an ultrafast $90^{\circ}$ polarization switch within the FFs pulse duration. Finally, for circularly polarized FFs, the emitted SH has opposite circular polarization due to valley-dependent selection rules (see the analysis of equation (4) $)^{44-46}$.

The SHG measurements were performed using the set-up shown in Fig. 2a and described in Methods. To realize all-optical polarization switching and $\mathrm{SH}$ modulation, it is crucial to first characterize the relative orientation between the FFs and the $\mathrm{MoS}_{2}$ sample. To do so, we first performed SHG experiments using a tunable near-infrared optical parametric oscillator (OPO) as the FF. The emitted SHG power for the FF wavelengths used in our experiments (between $1,360 \mathrm{~nm}$ and $1,560 \mathrm{~nm}$ ) is shown in Fig. 2b. The slope of 2 in the double logarithmic plot is further proof of a genuine SHG process. The crystal orientation of the $\mathrm{MoS}_{2}$ sample was determined for each FF wavelength by $\mathrm{SH}$ polarization-dependent experiments, with an extra polarizer in front of the detector to measure the $\mathrm{SH}$ 


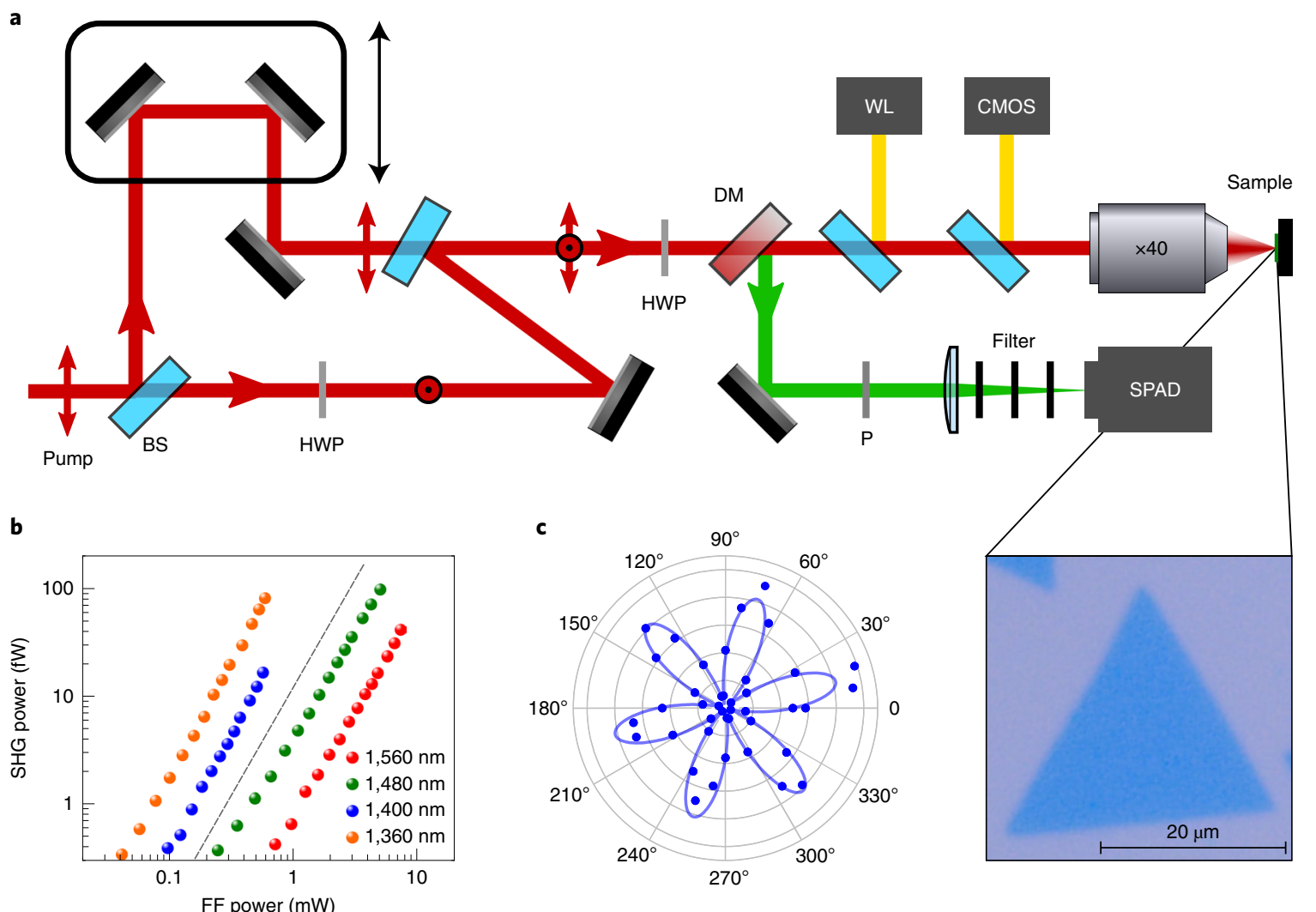

Fig. 2 | All-optical modulation set-up and SHG characterization. a, A sketch of the set-up used for the experiments. For the FF we used an OPO tunable between $\sim 1.3 \mu \mathrm{m}$ and $2.0 \mu \mathrm{m}$. The FF beams are separated into two perpendicular replicas inside of a Mach-Zehnder interferometer and subsequently focused onto the $\mathrm{MoS}_{2}$ sample with a $\times 40$ microscope objective. The backscattered SH signal is spectrally filtered and detected with a SPAD. CMOS, complementary metal-oxide semiconductor camera; BS, beam splitter; P, polarizer; HWP, half-wave plate; WL, white light. DM, dichroic mirror. b, The power dependence of the SHG signal for all wavelengths used in our experiments. The grey dashed line is a guide to the eye to indicate a slope of 2 , which is typical of the SHG process. c, A polar plot of the normalized SH intensity as a function of the excitation polarization angle $\theta$ with $\mathrm{SH}$ polarization detection always parallel to the FF. Blue circles show experimental data and the solid blue line indicates the $\cos ^{2}\left[3\left(\theta-\phi_{0}\right)\right]$ fit.

parallel to the excitation polarization ${ }^{36,39,43,47}$ : the $\mathrm{SH}$ intensity is proportional to $\cos ^{2}\left[3\left(\theta-\phi_{0}\right)\right]$, where $\theta$ is the FF polarization angle and $\phi_{0}$ is the rotation of the $\mathrm{AC}$ direction relative to the p-polarization in the laboratory frame. Figure $2 \mathrm{c}$ is an example of the $\mathrm{SH}$ polar plot for a FF wavelength of $1,400 \mathrm{~nm}$ and shows that the AC direction is tilted by $\phi_{0}=13.6^{\circ}+n \times 60^{\circ}$ (where $n$ is an integer) with respect to p-polarization in the laboratory coordinates. Furthermore, Fig. 1c confirms the absence of any detectable strain in our sample, as uniaxial strain would result in a symmetric attenuation along its direction of action ${ }^{38,39}$. The small asymmetry of the lobes along the two AC directions $\left(\sim 15^{\circ} / 70^{\circ}\right.$ and $\left.\sim 190^{\circ} / 250^{\circ}\right)$ is attributed to polarization-scrambling effects due to the use of a dichroic mirror in reflection geometry ${ }^{48}$. Finally, on the basis of the results in Fig. 2b, we determine the modulus of the complex second-order nonlinear susceptibility at the FF wavelengths used in our experiments. To do so, we estimate the optical losses of the set-up from the $\mathrm{SH}$ emission at the sample position to the detection on the single-photon avalanche diode (SPAD) and calculate the $\mathrm{SH}$ tensor element $\chi^{(2)}$ of $\mathrm{MoS}_{2}$, as described in Methods. We thus obtained effective second-order susceptibility values for our FF wavelengths at $1,360 \mathrm{~nm}, 1,400 \mathrm{~nm}, 1,480 \mathrm{~nm}$ and $1,560 \mathrm{~nm}$ of $\sim 282.1 \mathrm{pm} \mathrm{V}^{-1}, \sim 15$ $3.7 \mathrm{pm} \mathrm{V}^{-1}, \sim 44.7 \mathrm{pm} \mathrm{V}^{-1}$ and $\sim 24.2 \mathrm{pm} \mathrm{V}^{-1}$, respectively. The highest value obtained at $1,360 \mathrm{~nm}$ FF wavelength is due to exciton resonant $\mathrm{SH}$ enhancement ${ }^{15,49}$. All values are in good agreement with those previously reported by experiments performed at similar FF wavelength $\mathrm{s}^{36,42,49-51}$ and predicted by theor $\mathrm{y}^{52,53}$. It is worth noting that for single-layer TMDs where interlayer interference ${ }^{54}$ is absent, SHG is insensitive to the phase of the nonlinear optical response.

\section{Nonlinear all-optical modulation}

Having defined the AC and ZZ directions of our sample, we now demonstrate all-optical $\mathrm{SH}$ polarization and amplitude modulation. We separate the FF beam into two perpendicular replicas, align them along the $\mathrm{AC}$ and $\mathrm{ZZ}$ directions of the sample using a half-waveplate, and control their relative delay with a motorized mechanical stage (see Methods for details). For large delays (that is, longer than the FF pulse duration) between the two perpendicular FFs, SH will be emitted by each individual FF along the AC direction following equation (2). At zero delay (when the two FFs overlap perfectly in time) the $\mathrm{SH}$ intensity along the $\mathrm{AC}$ direction will go to zero and the SH signal will be emitted only along the $\mathrm{ZZ}$ direction. Figure 3 a shows the $\mathrm{SH}$ average power emitted along the $\mathrm{ZZ}$ direction as a function of the delay between the two perpendicularly polarized FFs and for a FF wavelength of $1,480 \mathrm{~nm}$. The Gaussian fit (the blue curve in Fig. 3a) has a full-width at half-maximum (FWHM) of $\sim 250 \mathrm{fs}$, which corresponds to the autocorrelation function of our OPO pulse with a duration of $\sim 175 \mathrm{fs}$. Moreover, the $\mathrm{SH}$ signal along the $\mathrm{ZZ}$ direction is now ideally background free, demonstrating the potential of ultrafast $\mathrm{SH}$ polarization switching and the close-to- $100 \%$ amplitude modulation of our approach.

We further note that this result is solely based on symmetry considerations and thus provides an ultrabroadband response that is 

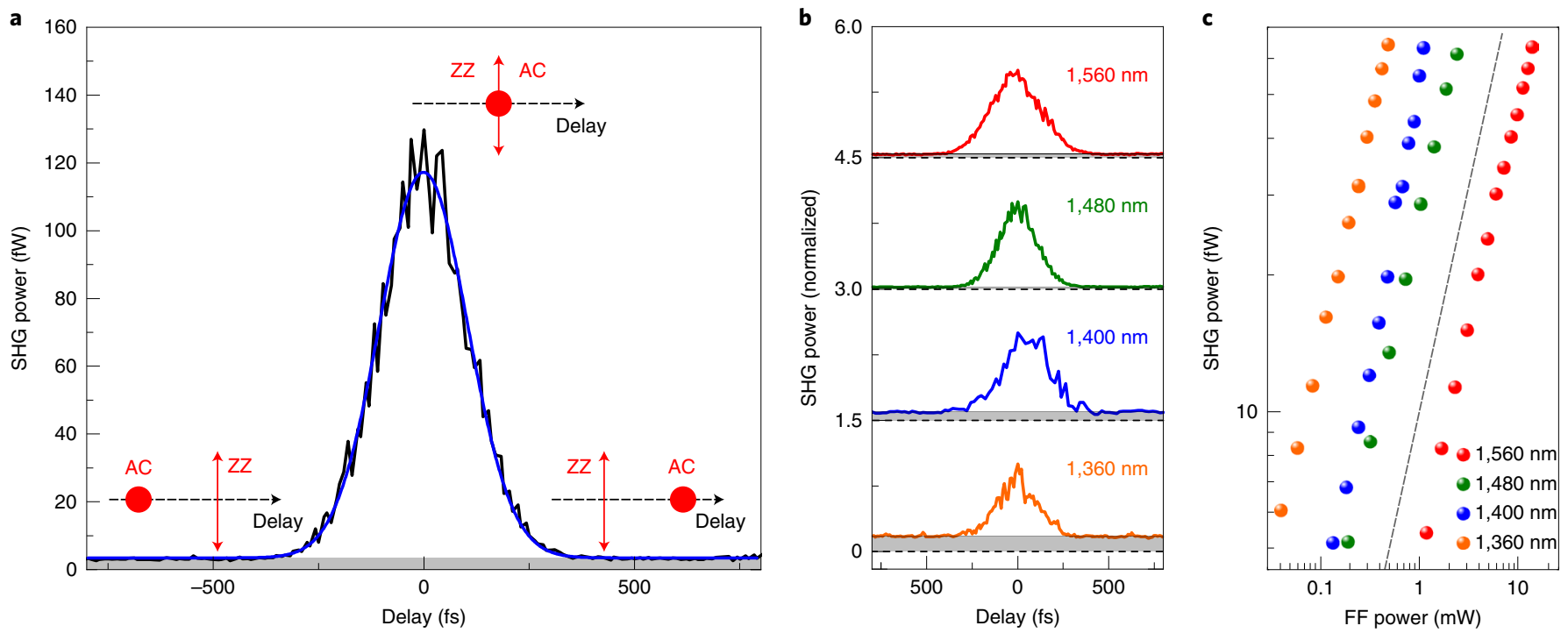

Fig. 3 Ultrafast polarization and amplitude all-optical switching. a, Second-harmonic intensity for an FF wavelength of 1,480 nm (black line) measured along the ZZ direction as a function of the delay between the two perpendicularly polarized FFs (illustrated in the inset). The blue line corresponds to a Gaussian fit of the autocorrelation curve, whereas the grey shaded area represents the noise level at $\sim 3.5 \mathrm{fW}$ of our experiments. $\mathbf{b}$, The normalized $\mathrm{SH}$ intensities and noise level for all of the FF wavelengths used in our experiments. c, A double logarithmic plot of the emitted SH power as a function of the incident FF power along the AC direction. The grey dashed line is a guide to the eye, representing a slope of 1 .

not limited to above-gap or resonant exciton pumping. We obtained the same result for all of the FF wavelengths used in our experiments, as shown in Fig. 3b. The possibility to emit SH along perpendicular directions ( $\mathrm{AC}$ and $\mathrm{ZZ}$ ) with the same efficiency is a unique feature that arises from the combination of symmetry and deep subwavelength thickness of TMDs, which relaxes the phase-matching constraints typical of harmonic generation. This result could have an immediate application in background-free ultrabroadband and ultrashort pulse characterization. For instance, in the most advanced commercial systems ${ }^{55}$ for ultrashort pulse characterization, one has to switch between collinear and non-collinear geometries to collect either the interferometric or the background-free intensity autocorrelation signals, respectively. By contrast, in our approach both signals are accessible using the same geometry and by simply switching the SH detection from AC to ZZ. Further, following equation (3), the power scaling of the emitted $\mathrm{SH}$ along the $\mathrm{ZZ}$ direction is linear with respect to each of the FF intensities. This is confirmed by the power-dependent SHG measurements reported in Fig. 3c, where we show the emitted $\mathrm{SH}$ power along the $\mathrm{ZZ}$ direction at zero delay between the two FFs and as a function of the AC-polarized FF power.

To gain more insight into the temporal evolution of the emitted $\mathrm{SH}$ polarization and amplitude, we scan the delay between the two perpendicularly polarized FFs with interferometric precision and measure the emitted $\mathrm{SH}$ along both the $\mathrm{AC}$ and $\mathrm{ZZ}$ directions (Fig. 4a). To control the delay between two perpendicular pulses with the desired sub-optical-cycle precision, we used the common-path delay generator sketched in Fig. $4 \mathrm{~b}$ and described in Methods. As expected, the $\mathrm{SH}$ power is emitted only along the AC direction for delays longer than our pulse duration, and no signal is detected along the ZZ direction (Fig. 4a). Instead, for delays close to zero we observe a strong ultrafast modulation of the $\mathrm{SH}$ power emitted along the AC direction. This can be better appreciated by looking at Fig. $4 \mathrm{c}$, which shows the emitted SH power along the AC and ZZ directions at $1,480 \mathrm{~nm}$ for delays between $-10 \mathrm{fs}$ and $+10 \mathrm{fs}$.

It is useful to note that, for delays much shorter than the pulse duration, our interferometric measurement is the analogue of tuning the polarization of one FF pulse along the orthodrome of the Poincaré sphere (see the inset in Fig. 4a). This corresponds to a rotation of the FF polarization from $-45^{\circ}$ with respect to the $\mathrm{AC}-\mathrm{ZZ}$ directions (at zero delay), to left/right circular polarization (at $\tau= \pm \frac{T}{4}$ delay, where $\tau$ is the FF optical cycle), to $+45^{\circ}$ with respect to the AC-ZZ directions (at $\tau= \pm \frac{T}{2}$ delay). This result is consistent with the theoretical SH polarization $\mathbf{P}^{(2)}$ generated by an arbitrary elliptically polarized $\mathrm{FF}^{46}$ after a simple basis transformation to account for the rotation by $-45^{\circ}$ with respect to AC/ZZ directions:

$$
\mathbf{P}^{(2)}=\epsilon_{0} \chi^{(2)}|\mathbf{E}|^{2}(\widehat{\mathrm{ZZ}} \pm i \sin (2 \vartheta) \widehat{\mathrm{AC}})
$$

Here $\vartheta=0^{\circ}$ denotes a linearly polarized $\mathrm{FF}$ at $45^{\circ}$ with respect to the AC/ZZ direction, whereas $\vartheta=45^{\circ}$ corresponds to a circularly polarized FF. This clearly shows that the SH component emitted along the AC direction oscillates with a period of $\frac{T}{2}$ as a function of the FF polarization, by contrast to the $\mathrm{SH}$ emitted along the $\mathrm{ZZ}$ direction. This underpins the interferometric precision required to fully capture the modulation along the AC direction. The experimental results show a weak modulation also for the $\mathrm{SH}$ emitted along the ZZ direction, although this is not expected from theory. This could arise from weak strain (that is, below the limit detectable by our SHG polarization measurements ${ }^{39,56}$ ), small deviations in the alignment of the detection polarizer with respect to the $\mathrm{AC} / \mathrm{ZZ}$ directions or from extra terms in the $\chi^{(2)}$ arising from the valley degree of freedom ${ }^{48,57}$. Looking at Fig. $4 \mathrm{c}$, one can indeed appreciate that the $\mathrm{SH}$ is emitted only along the $\mathrm{ZZ}$ direction at zero delay ( $\mathrm{FF}$ at $-45^{\circ}$ with respect to $\mathrm{AC} / \mathrm{ZZ}$ directions), whereas the emitted $\mathrm{SH}$ components along $\mathrm{AC}$ and $\mathrm{ZZ}$ are identical at $\frac{T}{4}$ delay, as expected for circular polarization.

\section{Discussion}

In conclusion, we have demonstrated all-optical polarization switching and amplitude modulation of SHG in $\mathrm{MoS}_{2}$. Our approach surpasses all previously reported electrical and all-optical attempts of $\mathrm{SH}$ tuning in terms of modulation depth and speed, providing a $90^{\circ}$ polarization switch, modulation depth close to $100 \%$, and speed 
a

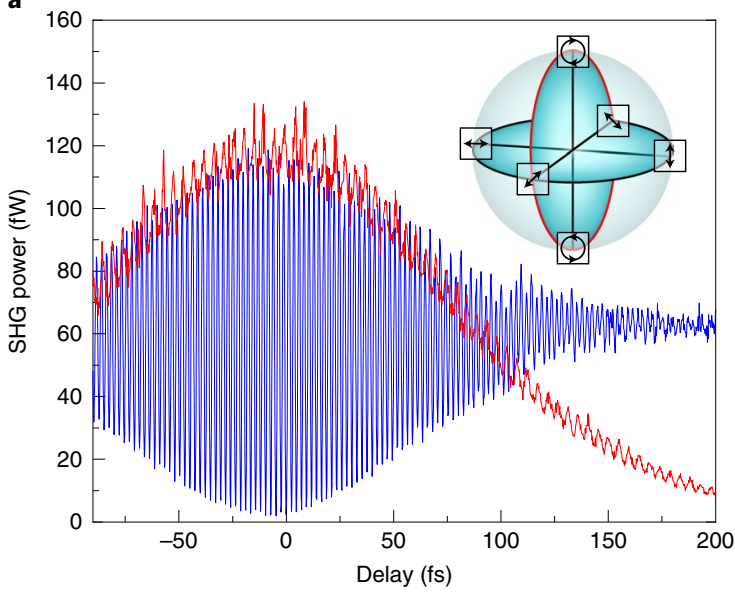

b

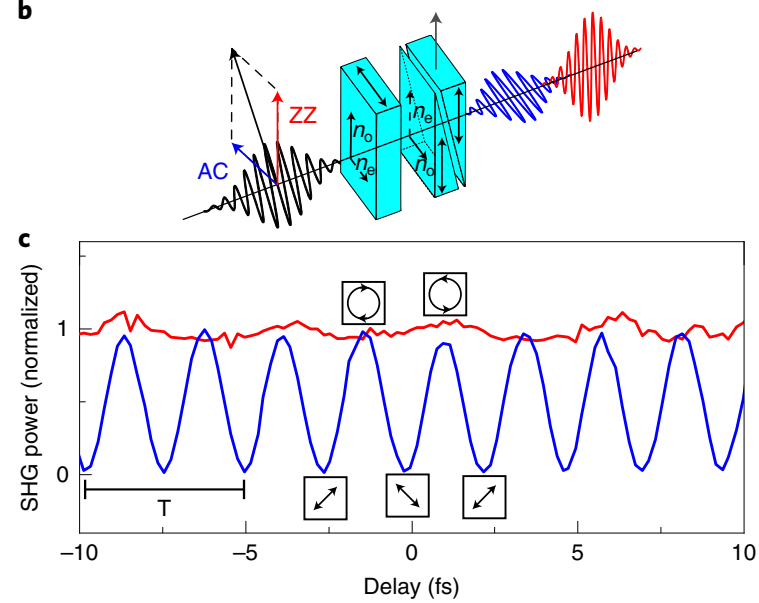

Fig. 4 | Phase-locked all-optical SH modulation along the AC and ZZ directions. a, Second-harmonic power for an FF wavelength of 1,480 nm along the AC (blue) and ZZ (red) directions as a function of the relative delay between the two perpendicularly polarized FFs. The inset shows the Poincaré sphere with polarization directions on its orthodromes. The red line indicates the change in polarization when tuning the delay between the two FFs with a birefringent delay line. $\mathbf{b}$, A schematic of the modified common-path delay generator. The two perpendicular components of an incident pulse-polarized at $45^{\circ}$ with respect to the $\mathrm{AC} / \mathrm{ZZ}$ directions-are delayed by two birefringent alpha barium borate crystals with a perpendicular optical axis, whereby the variable thickness of the second one allows to control the delay with interferometric precision. $\mathbf{c}, \mathrm{A}$ magnification of $\mathbf{a}$, showing the detected $\mathrm{SH}$ power for a 1,480 nm FF along the AC (blue) and ZZ (red) directions close to zero delay.

limited only by the FF pulse duration. Moreover, our method is intrinsically broadband as it only relies on the crystal symmetry of TMDs. We thus foresee a direct impact of our results on a variety of photonic devices, such as high-speed frequency converters, nonlinear all-optical modulators and transistors ${ }^{31,32}$, autocorrelators for ultrashort pulse characterization, and atomically thin optically tunable nonlinear holograms ${ }^{24}$.

\section{Online content}

Any methods, additional references, Nature Research reporting summaries, source data, extended data, supplementary information, acknowledgements, peer review information; details of author contributions and competing interests; and statements of data and code availability are available at https://doi.org/10.1038/ s41566-021-00859-y.

Received: 7 April 2021; Accepted: 12 July 2021; Published online: 9 September 2021

\section{References}

1. Franken, P. A., Hill, A. E., Peters, C. W. \& Weinreich, G. Generation of optical harmonics. Phys. Rev. Lett. 7, 118-119 (1961).

2. Hickstein, D. D. et al. Self-organized nonlinear gratings for ultrafast nanophotonics. Nat. Photon. 13, 494-499 (2019).

3. Schmitt, T. et al. Control of crystal symmetry breaking with halogen-substituted benzylammonium in layered hybrid metal-halide perovskites. J. Am. Chem. Soc. 142, 5060-5067 (2020).

4. Frohna, K. et al. Inversion symmetry and bulk Rashba effect in methylammonium lead iodide perovskite single crystals. Nat. Commun. $\mathbf{9}$, 1829 (2018)

5. Tran, R. J., Sly, K. L. \& Conboy, J. C. Applications of surface second harmonic generation in biological sensing. Ann. Rev. Anal. Chem. 10, 387-414 (2017).

6. Shen, Y. Optical second harmonic generation at interfaces. Ann. Rev. Phys. Chem. 40, 327-350 (1989).

7. Weiner, A. Ultrafast Optics Vol. 72 (Wiley, 2011).

8. Wang, C. et al. Metasurface-assisted phase-matching-free second harmonic generation in lithium niobate waveguides. Nat. Commun. 8, 2098 (2017).

9. Chen, J.-Y. et al. Ultra-efficient frequency conversion in quasi-phase-matched lithium niobate microrings. Optica 6, 1244-1245 (2019).

10. Cazzanelli, M. et al. Second-harmonic generation in silicon waveguides strained by silicon nitride. Nat. Mater. 11, 148-154 (2012).
11. Timurdogan, E., Poulton, C. V., Byrd, M. \& Watts, M. Electric field-induced second-order nonlinear optical effects in silicon waveguides. Nat. Photon. 11, 200-206 (2017).

12. Lu, X., Moille, G., Rao, A., Westly, D. A. \& Srinivasan, K. Efficient photoinduced second-harmonic generation in silicon nitride photonics. Nat. Photon. 15, 131-136 (2021).

13. Autere, A. et al. Nonlinear optics with $2 \mathrm{D}$ layered materials. Adv. Mater. 30, 1705963 (2018).

14. Trovatello, C. et al. Optical parametric amplification by monolayer transition metal dichalcogenides. Nat. Photon. 15, 6-10 (2021).

15. Wang, G. et al. Giant enhancement of the optical second-harmonic emission of $\mathrm{WSe}_{2}$ monolayers by laser excitation at exciton resonances. Phys. Rev. Lett. 114, 097403 (2015).

16. Soavi, G. et al. Broadband, electrically tunable third-harmonic generation in graphene. Nat. Nanotechnol. 13, 583-588 (2018).

17. Massicotte, M. et al. Hot carriers in graphene-fundamentals and applications. Nanoscale 13, 8376-8411 (2021).

18. Ferrari, A. C. et al. Science and technology roadmap for graphene, related two-dimensional crystals, and hybrid systems. Nanoscale 7, 4598-4810 (2015).

19. An, N. et al. Electrically tunable four-wave-mixing in graphene heterogeneous fiber for individual gas molecule detection. Nano Lett. 20, 6473-6480 (2020).

20. Alexander, K., Savostianova, N. A., Mikhailov, S. A., Kuyken, B. \& Van Thourhout, D. Electrically tunable optical nonlinearities in graphene-covered sin waveguides characterized by four-wave mixing. ACS Photon. 4, 3039-3044 (2017)

21. Liu, T. et al. Low-loss integrated nanophotonic circuits with layered semiconductor materials. Nano Lett. 21, 2709-2718 (2021).

22. van de Groep, J. et al. Exciton resonance tuning of an atomically thin lens. Nat. Photon. 14, 426-430 (2020).

23. Lin, H. et al. Diffraction-limited imaging with monolayer 2D material-based ultrathin flat lenses. Light Sci. Appl. 9,137 (2020).

24. Dasgupta, A., Gao, J. \& Yang, X. Atomically thin nonlinear transition metal dichalcogenide holograms. Nano Lett. 19, 6511-6516 (2019).

25. Löchner, F. J. et al. Controlling second-harmonic diffraction by nano-patterning $\mathrm{MoS}_{2}$ monolayers. Opt. Exp. 27, 35475-35484 (2019).

26. Seyler, K. L. et al. Electrical control of second-harmonic generation in a $\mathrm{WSe}_{2}$ monolayer transistor. Nat. Nanotechnol. 10, 407-411 (2015).

27. Klein, J. et al. Electric-field switchable second-harmonic generation in bilayer $\mathrm{MoS}_{2}$ by inversion symmetry breaking. Nano Lett. 17, 392-398 (2017).

28. Soavi, G. et al. Hot electrons modulation of third-harmonic generation in graphene. ACS Photon. 6, 2841-2849 (2019).

29. Taghinejad, M. et al. Photocarrier-induced active control of second-order optical nonlinearity in monolayer $\mathrm{MoS}_{2}$. Small 16, 1906347 (2020).

30. Cheng, Y. et al. Ultrafast optical modulation of harmonic generation in two-dimensional materials. Nano Lett. 20, 8053-8058 (2020). 
31. Wang, Z., Hagan, D., Van Stryland, E. \& Assanto, G. Second harmonic generation: toward an all-optical transistor. Opt. Photon. News 6, 13-14 (1995).

32. Mingaleev, S. \& Kivshar, Y. Nonlinear photonic crystals toward all-optical technologies. Opt. Photon. News 13, 48-51 (2002).

33. George, A. et al. Controlled growth of transition metal dichalcogenide monolayers using Knudsen-type effusion cells for the precursors. J. Phys. Mater. 2, 016001 (2019).

34. Shree, S. et al. High optical quality of $\mathrm{MoS}_{2}$ monolayers grown by chemical vapor deposition. 2D Mater. 7, 015011 (2019).

35. Boyd, R. W. Nonlinear Optics (Academic, 2020).

36. Malard, L. M., Alencar, T. V., Barboza, A. P. M., Mak, K. F. \& De Paula, A. M. Observation of intense second harmonic generation from $\mathrm{MoS}_{2}$ atomic crystals. Phys. Rev. B 87, 201401 (2013).

37. Kumar, N. et al. Second harmonic microscopy of monolayer $\mathrm{MoS}_{2}$. Phys. Rev. B 87, 161403 (2013).

38. Mennel, L. et al. Optical imaging of strain in two-dimensional crystals. Nat. Commun. 9, 516 (2018).

39. Mennel, L., Paur, M. \& Müller, T. Second harmonic generation in strained transition metal dichalcogenide monolayers: $\mathrm{MoS}_{2}, \mathrm{MoSe}_{2}, \mathrm{WS}_{2}$, and $\mathrm{WSe}_{2}$. APL Photon. 4, 034404 (2019).

40. Mennel, L. et al. Band nesting in two-dimensional crystals: an exceptionally sensitive probe of strain. Nano Lett. 20, 4242-4248 (2020).

41. Wen, X., Gong, Z. \& Li, D. Nonlinear optics of two-dimensional transition metal dichalcogenides. InfoMat 1, 317-337 (2019).

42. Woodward, R. et al. Characterization of the second-and third-order nonlinear optical susceptibilities of monolayer $\mathrm{MoS}_{2}$ using multiphoton microscopy. 2D Mater. 4, 011006 (2016).

43. Rosa, H. G. et al. Characterization of the second-and third-harmonic optical susceptibilities of atomically thin tungsten diselenide. Sci. Rep. 8, 10035 (2018).

44. Zhang, D. et al. Near-unity polarization of valley-dependent second-harmonic generation in stacked TMDC layers and heterostructures at room temperature. Adv. Mater. 32, 1908061 (2020).

45. Xiao, J. et al. Nonlinear optical selection rule based on valley-exciton locking in monolayer $\mathrm{WS}_{2}$. Light Sci. Appl. 4, e366 (2015).

46. Säynätjoki, A. et al. Ultra-strong nonlinear optical processes and trigonal warping in $\mathrm{MoS}_{2}$ layers. Nat. Commun. 8, 1-8 (2017).

47. Li, Y. et al. Probing symmetry properties of few-layer $\mathrm{MoS}_{2}$ and h-bn by optical second-harmonic generation. Nano Lett. 13, 3329-3333 (2013).
48. Mouchliadis, L. et al. Probing valley population imbalance in transition metal dichalcogenides via temperature-dependent second harmonic generation imaging. npj 2D Mater. Appl. 5, 1-9 (2021).

49. Le, C. T. et al. Impact of selenium doping on resonant second-harmonic generation in monolayer $\mathrm{MoS}_{2}$. ACS Photon. 4, 38-44 (2017).

50. Clark, D. et al. Near bandgap second-order nonlinear optical characteristics of $\mathrm{MoS}_{2}$ monolayer transferred on transparent substrates. Appl. Phys. Lett. 107, 131113 (2015).

51. da Fonseca, L. L. P. et al. Second- and third-order optical susceptibilities across excitons states in $2 \mathrm{D}$ monolayer transition metal dichalcogenides. 2D Mater. 8, 035010 (2021).

52. Trolle, M. L., Seifert, G. \& Pedersen, T. G. Theory of excitonic second-harmonic generation in monolayer $\mathrm{MoS}_{2}$. Phys. Rev. B 89, 235410 (2014).

53. Clark, D. et al. Strong optical nonlinearity of CVD-grown $\mathrm{MoS}_{2}$ monolayer as probed by wavelength-dependent second-harmonic generation. Phys. Rev. B 90, 121409 (2014).

54. Kim, W., Ahn, J. Y., Oh, J., Shim, J. H. \& Ryu, S. Second-harmonic Young's interference in atom-thin heterocrystals. Nano Lett. 20, 8825-8831 (2020)

55. pulseCheck. APE https://www.ape-berlin.de/en/autocorrelator/pulsecheck/ (2021).

56. Liang, J. et al. Monitoring local strain vector in atomic-layered $\mathrm{MoSe}_{2}$ by second-harmonic generation. Nano Lett. 17, 7539-7543 (2017).

57. Ho, Y. W. et al. Measuring valley polarization in two-dimensional materials with second-harmonic spectroscopy. ACS Photon. 7, 925-931 (2020).

Publisher's note Springer Nature remains neutral with regard to jurisdictional claims in published maps and institutional affiliations.

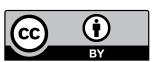

Open Access This article is licensed under a Creative Commons

Attribution 4.0 International License, which permits use, sharing, adaptation, distribution and reproduction in any medium or format, as long as you give appropriate credit to the original author(s) and the source, provide a link to the Creative Commons license, and indicate if changes were made. The images or other third party material in this article are included in the article's Creative Commons license, unless indicated otherwise in a credit line to the material. If material is not included in the article's Creative Commons license and your intended use is not permitted by statutory regulation or exceeds the permitted use, you will need to obtain permission directly from the copyright holder. To view a copy of this license, visit http://creativecommons. org/licenses/by/4.0/.

(c) The Author(s) 2021 


\section{Methods}

Polarization-dependent SH intensity. The vectorial components of the second-order polarization $P^{(2)}(2 \omega)$ for a material with $D_{3 h}$ symmetry (such as TMDs) are given by

$$
\left(\begin{array}{l}
P_{x}^{(2)}(2 \omega) \\
P_{y}^{(2)}(2 \omega) \\
P_{z}^{(2)}(2 \omega)
\end{array}\right)=\epsilon_{0}\left(\begin{array}{cccccc}
0 & 0 & 0 & 0 & 0 & \chi_{x x y}^{(2)} \\
\chi_{y x x}^{(2)} & \chi_{y y y}^{(2)} & 0 & 0 & 0 & 0 \\
0 & 0 & 0 & 0 & 0 & 0
\end{array}\right)\left(\begin{array}{l}
E_{x}^{2}(\omega) \\
E_{y}^{2}(\omega) \\
E_{z}^{2}(\omega) \\
2 E_{y}(\omega) E_{z}(\omega) \\
2 E_{x}(\omega) E_{z}(\omega) \\
2 E_{x}(\omega) E_{y}(\omega)
\end{array}\right)
$$

where $\chi_{y y y}^{(2)}=-\chi_{x x y}^{(2)}=-\chi_{y x x}^{(2)}=\chi^{(2)}$. If we now consider a TMD oriented in such way that the ZZ and AC directions lie along the $x$ and $y$ Cartesian coordinates, respectively, and we neglect the $z$ (out-of-plane) direction, we obtain the following expression:

$$
\left(\begin{array}{l}
P_{\mathrm{ZZ}}^{(2)}(2 \omega) \\
P_{\mathrm{AC}}^{(2)}(2 \omega)
\end{array}\right)=\epsilon_{0} \chi^{(2)}(2 \omega, \omega, \omega)\left(\begin{array}{l}
2 E_{\mathrm{ZZ}}(\omega) E_{\mathrm{AC}}(\omega) \\
E_{\mathrm{ZZ}}^{2}(\omega)-E_{\mathrm{AC}}^{2}(\omega)
\end{array}\right)
$$

Finally, as the $\mathrm{SH}$ intensity is proportional to the absolute square value of the second-order polarization, we retrieve equations (2) and (3) shown in the main text:

$$
\begin{aligned}
& I_{\mathrm{AC}}^{2 \omega}=\left|P_{\mathrm{AC}}^{(2)}\right|^{2} \propto\left|E_{\mathrm{AC}}^{2}-E_{\mathrm{ZZ}}^{2}\right|^{2} \\
& I_{\mathrm{ZZ}}^{2 \omega}=\left|P_{\mathrm{ZZ}}^{(2)}\right|^{2} \propto\left|2 E_{\mathrm{AC}} E_{\mathrm{ZZ}}\right|^{2}
\end{aligned}
$$

SHG set-up. For the FF we used an OPO (Levante IR from APE) pumped by an ytterbium-doped mode locked laser (FLINT12, Light Conversion) with a repetition rate of $76 \mathrm{MHz}$, pulse duration of $100 \mathrm{fs}$, and generating pulses tunable between $\sim 1.3 \mu \mathrm{m}$ and $2.0 \mu \mathrm{m}$. The FF is then separated into two perpendicular replicas whose relative delay is tuned with two different approaches: a computer-controlled motorized stage (M-414.2PD, PI) in a Mach-Zehnder interferometer configuration and a commercial common-path birefringent interferometer (GEMINI, NIREOS) ${ }^{58}$. Compared with standard home-built interferometers, the GEMINI provides subwavelength interferometric stability with precise control on the delay between the two replicas with attosecond precision. The polarization of the FFs was tuned using a half-waveplate (AHWP05M-1600, Thorlabs) and the power on the sample was controlled by two polarizers (LPNIR050 and WP12L-UB, both Thorlabs). Finally, the two collinear and perpendicularly polarized FFs were focused on the sample using a custom built microscope equipped with a $\times 40$ reflective objective (LMM-40X-P01, Thorlabs). The backscattered SH signal is spectrally separated using a dichroic mirror (DMSP950, Thorlabs), further spectrally purified by filters (FELH0650, FESH850, FESH0950, all Thorlabs) and detected with a SPAD (C11202-050, Hamamatsu). The $\mathrm{SH}$ polarization was measured using a wire grid polarizer (WP12L-UB, Thorlabs).

Estimate of the optical losses of the set-up. To quantify the SH signal generated directly at the sample position, optical losses of the different components of the set-up must be considered. Although the transmission coefficients for the investigated SH wavelengths of the filters and the dichroic mirror (all >96\%) were taken from the manufacturer's website, the values for polarizers and the microscope objective were determined experimentally. A transmission of $~ 79 \%$ was determined for the wire grid polarizer, whereas we determined a transmission of $50 \%$ for the reflective objective. Last, the responsivity of the SPAD was taken into account, which ranges depending on the investigated $\mathrm{SH}$ wavelength between $\sim 17 \%$ and $\sim 31 \%$. In total, we estimated our optical losses from the SH emission to the detector to be $\sim 86-92 \%$, depending on the wavelength.

Calculation of the second-order nonlinear susceptibility. The sheet $\mathrm{SH}$ tensor element $\chi_{\mathrm{S}}^{(2)}$ can be calculated from the FF and SH average powers using the equation ${ }^{42}$ :

$$
\chi_{\mathrm{S}}^{(2)}=\sqrt{\frac{c^{3} \epsilon_{0} f \pi r^{2} t_{\mathrm{FWHM}}\left(1+n_{2}\right)^{6} P_{\mathrm{SHG}}(2 \omega)}{16 \sqrt{2} S \omega^{2} P_{\mathrm{FF}}^{2}(\omega)}},
$$

where $c$ is the speed of light, $\epsilon_{0}$ is the permittivity of free-space, $f=76 \mathrm{MHz}$ is the pump laser repetition rate, $r \approx 1.85 \mu \mathrm{m}$ is the focal spot radius, $t_{\mathrm{FWHM}} \approx 200 \mathrm{fs}$ is the FWHM of the pulse, $n_{2} \approx 1.45$ is the substrate refractive index, $S=0.94$ is a shape factor for Gaussian pulses, $\omega$ is the FF angular frequency, and $P_{\mathrm{SHG}}(2 \omega)-P_{\mathrm{FF}}(\omega)$ are the $\mathrm{SH}$ and FF average powers, respectively. The effective bulk-like second-order susceptibility $\chi_{\text {eff }}^{(2)}$ can be subsequently calculated from equation (5) as $\chi_{\text {eff }}^{(2)}=\frac{\chi_{s}^{(2)}}{d_{\mathrm{Mos}_{2}}}$, where the thickness of $\mathrm{MoS}_{2} d_{\mathrm{MoS}_{2}}$ is $0.65 \mathrm{~nm}$ (refs. ${ }^{18,46}$ ).

Pulse duration of the FFs. To prove that our method is solely limited by the pulse duration of the FFs, we performed a standard characterization of a temporal profile of our OPO source at different wavelengths (Extended Data Fig. 1). For the measurements, we used a home-built autocorrelator based on a Michelson interferometer equipped with a motorized and computer-controlled translation stage (HPS60-20X-M5, Optosigma). Two identical and temporally delayed replicas of the OPO pulse were then focused onto a 1-mm-thick beta barium borate crystal (BBO-652H, Eksma Optics) in non-collinear geometry and the background-free SHG autocorrelation intensity was detected on a silicon photodetector (DET10A2, Thorlabs). From this we obtained values for the autocorrelation in the range of $217 \mathrm{fs}$ to $310 \mathrm{fs}$ with Gaussian fits, corresponding to pulse durations between $150 \mathrm{fs}$ and $220 \mathrm{fs}$, respectively.

\section{Data availability}

The data that support the plots within this paper and other findings of this study are available from the corresponding author on reasonable request. Source data are provided with this paper

\section{References}

58. Preda, F. et al. Linear and nonlinear spectroscopy by a common-path

birefringent interferometer. IEEE J. Sel. Top. Quant. Electron. 23, 88-96 (2016).

\section{Acknowledgements}

We acknowledge H. Rostami and F. Preda for helpful discussions. This work was supported by the European Union's Horizon 2020 Research and Innovation programme under Grant Agreement GrapheneCore3 881603 (G.S. and G.C.). This publication is part of the METAFAST project that received funding from the European Union's Horizon 2020 Research and Innovation programme under Grant Agreement No. 899673 (G.S. and G.C.). We acknowledge the German Research Foundation DFG (CRC 1375 NOA project numbers B2 (A.T.) and B5 (G.S.)) and the Daimler und Benz foundation for financial support (G.S.). Open-access funding was provided by Friedrich-Schiller-Universität Jena.

\section{Author contributions}

S.K. and G.S. conceived the experiments. S.K. and O.G. performed the all-optical modulation measurements. Z.G., A.G. and A.T. fabricated and provided the high-quality $\mathrm{MoS}_{2}$ sample. S.K., G.C and G.S. wrote the manuscript, with contributions from all authors. All authors participated in the discussion and commented on the manuscript.

\section{Funding}

Open access funding provided by Friedrich-Schiller-Universität Jena (1010).

\section{Competing interests}

The authors declare no competing interests.

\section{Additional information}

Extended data is available for this paper at https://doi.org/10.1038/s41566-021-00859-y. Supplementary information The online version contains supplementary material available at https://doi.org/10.1038/s41566-021-00859-y.

Correspondence and requests for materials should be addressed to Giancarlo Soavi. Peer review information Nature Photonics thanks Goki Eda and the other, anonymous, reviewer(s) for their contribution to the peer review of this work.

Reprints and permissions information is available at www.nature.com/reprints. 


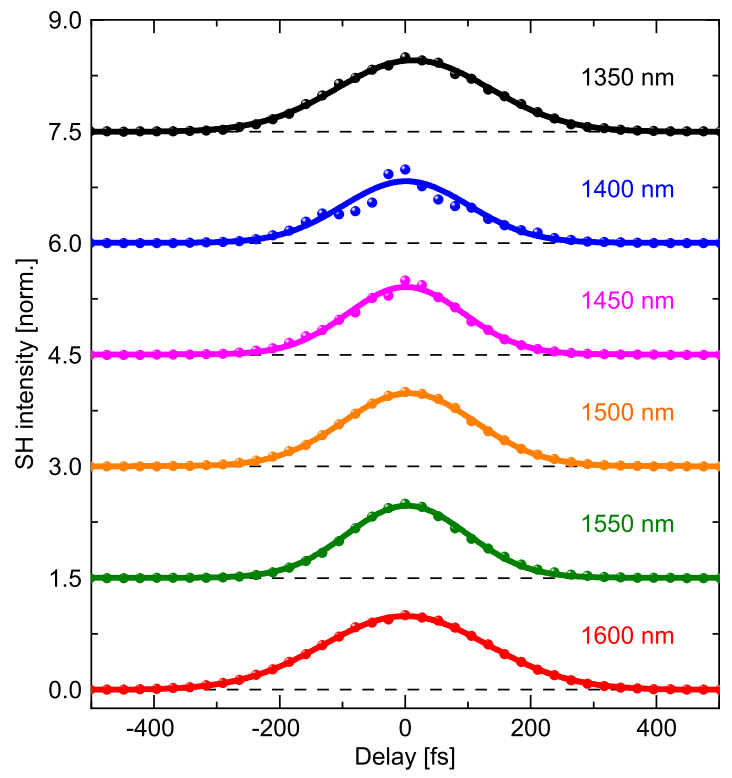

Extended Data Fig. 1 | Normalized SHG intensity autocorrelations of various OPO wavelengths in the investigated interval. Colored dots are for experimental data and solid lines are for gaussian fits. The plots are vertically shifted for clarity. 